\title{
Morphing Planar Graph Drawings with Bent Edges
}

\author{
Anna Lubiw ${ }^{* \dagger}$ and Mark Petrick* ${ }^{* \ddagger}$
}

\begin{abstract}
We give an algorithm to morph between two planar drawings of a graph, preserving planarity, but allowing edges to bend during the course of the morph. The morph uses a polynomial number of elementary steps, where each elementary step is a linear morph that moves each vertex in a straight line at uniform speed. Although there are planarity-preserving morphs that do not require edge bends, it is an open problem to find polynomial-size morphs. We achieve polynomial size at the expense of edge bends.
\end{abstract}

\section{Introduction}

A morph from one drawing of a planar graph to another is a continuous transformation from the first drawing to the second that maintains planarity. Developments in the theory of morphing run parallel to the developments in graph drawing, though they lag behind. In particular, the milestones in the history of planar graph drawing are: the existence results for straight line planar drawings due to Wagner, Fáry and Stein; Tutte's algorithm to construct such drawings; and, in 1990, the polynomial time algorithms of de Frayssiex, Pach, Pollack [2] and independently Schnyder [5] to construct a straight-line drawing of an $n$-vertex planar graph on an $O(n) \times O(n)$ grid.

Mirroring these, the first result on morphing planar graph drawings was an existence result: between any two planar straight-line drawings there exists a morph in which every intermediate drawing is straight-line planar. This was proved for triangulations, by Cairns [1] in 1944, and extended to planar graphs by Thomassen [6] in 1983. Both proofs are constructive - they work by repeatedly contracting one vertex to another. Unfortunately, they use an exponential number of steps, and are horrible for visualization purposes since the graph contracts to a triangle and then re-emerges.

The next development was an algorithm to morph between any two planar straightline drawings, given by Floater and Gotsman [3] in 1999 for triangulations, and extended to planar graphs by Gotsman and Surazhsky [4] in 2001. The morphs are not given by means of explicit vertex trajectories, but rather by means of "snapshots" of the graph at any intermediate time $t$. By choosing sufficiently many values of $t$, they give good visual results,

${ }^{*}$ David R. Cheriton School of Computer Science, University of Waterloo, Waterloo, ON, Canada, N2L $3 \mathrm{G} 1$

$\dagger$ alubiw@uwaterloo.ca

${ }^{\ddagger}$ mdtpetrick@uwaterloo.ca 
but there is no proof that polynomially many steps suffice. Furthermore, the morph suffers from the same drawbacks as Tutte's original planar graph drawing algorithm in that there is no nice bound on the size of the grid needed for the drawings.

The history of morphing planar graph drawings has not progressed to the analogue of the small grid results of de Fraysseix et al.: It is an open problem to find a polynomial size morph between two given drawings of a planar graph.

In this paper we solve this problem provided that edges are allowed to bend during the course of the morph. We give a polynomial-time algorithm to find a planarity preserving morph between two drawings of a planar graph on $n$ vertices, where the morph is composed of a sequence of $O\left(n^{6}\right)$ linear morphs. During the course of the morph, an edge becomes a polygonal path with $O\left(n^{5}\right)$ bends.

Terminology. A planar drawing of a graph $G=(V, E)$ assigns to each vertex $v \in V$ a distinct point $p(v)$ in the plane, and to each edge $e=(u, v)$ a path from $p(u)$ to $p(v)$ in such a way that paths intersect only at a common endpoint. A plane graph is one that has a planar drawing. A combinatorial or planar embedding of a plane graph is a cyclic order of edges around vertices consistent with a planar drawing. We will consider drawings in which edges are drawn as polygonal paths. A point where such a polygonal path changes direction is called a bend.

A morph from a drawing $P$ of a graph $G$ to a drawing $Q$ of the graph is a continuous family of drawings $P(t)$, indexed by time $t \in[0,1]$ where each $P(t)$ is a drawing of $G$, and $P(0)=P$ and $P(1)=Q$. A morph preserves planarity if each $P(t)$ is planar.

\section{The Morphing Algorithm}

We give an algorithm that takes two planar straight line drawings $P$ and $Q$ representing the same combinatorial embedding of a graph $G$, and finds a planarity-preserving edge-bending morph from $P$ to $Q$ using a polynomial number of elementary steps.

Conceptually, the morph is simple: if $v_{1}, v_{2}, \ldots, v_{n}$ are the vertices of $Q$ ordered by $x$ coordinate, then we first locate $v_{1}$ in $P$-it must be on the outer face -and "pull it out" of the drawing until it is at the far left, allowing the edges of $P$ to bend in compensation. We then repeat with $v_{2}, v_{3}$ and so on until the vertices of $P$ appear in the same $x$-ordering as those of $Q$. If the pulling out is done with care, the edges of $P$ will now be polygonal paths monotone in the $x$ direction. We then perform a linear morph to adjust all vertices and bends to the correct $y$ coordinate, thus straightening all the polygonal paths.

We now discuss the algorithm in more detail, beginning with the set-up phase. We discretize by adding a vertical line through each vertex of $P$. We also add $n$ vertical lines $L_{i}, 1 \leq i \leq n$ to the left of the drawing of $P$. Line $L_{i}$ will be the eventual home of vertex $v_{i}$, and when all vertices $v_{i}$ reach their line $L_{i}$ then the vertices of $P$ are in the same $x$-order as those of $Q$. We plan ahead of time the route that each vertex of $P$ will follow as we pull it to its eventual position at the left. Specifically, we augment $Q$ with an extra vertex $v_{0}$ at the far left, and augment with extra straight-line edges so that every vertex $v_{i}, 1 \leq i \leq n$ is joined by some edge $e_{i}$ to a vertex earlier in the ordering. We augment $P$ to match the augmented $Q$ and its embedding by routing each new edge as a polygonal path. 
The main body of the algorithm is an iteration on $i=1, \ldots, n$. In iteration $i$ vertex $v_{i}$ is pulled along the path of $e_{i}$ until it reaches line $L_{i}$. This is accomplished by repeatedly moving $v_{i}$ from the vertical line on which it lies to an adjacent vertical line along the last segment of $e_{i}$. We call this the main step of the algorithm, and give details below. Each main step is preceded by a straightening step that modifies the incoming edges to $v_{i}$. Incoming and outgoing edges to a vertex are defined wrt the $x$-ordering in $Q$. In $P$, the incoming edges need not come from the left but they are contiguous in the cyclic order of edges. The straightening step, whose details we defer to the full version of the paper, enforces:

Property 1. All incoming edges to $v_{i}$ enter $v_{i}$ from the same side of the vertical line $l$ through $v_{i}$. If $l^{\prime}$ is the adjacent vertical line on that side, there are no other vertices/bends along $l^{\prime}$ between the incoming edges.

The final phase of the algorithm is a linear morph from $P$ to $Q$. We prove that it preserves planarity by proving that, after the main body of the algorithm, the trapezoidizations of $P$ and $Q$ are combinatorially the same. Details are defered.

\subsection{Main Step of Algorithm}

In the main step of the algorithm vertex $v_{i}$ is moved from its current vertical line $l$ to an adjacent line $l^{\prime}$ along the path of edge $e_{i}$. This step takes place repeatedly during iteration $i$ of the algorithm. Iterations $1, \ldots, i-1$ have already moved vertices $v_{1}, \ldots, v_{i-1}$ to lines $L_{1}, \ldots, L_{i-1}$ respectively.
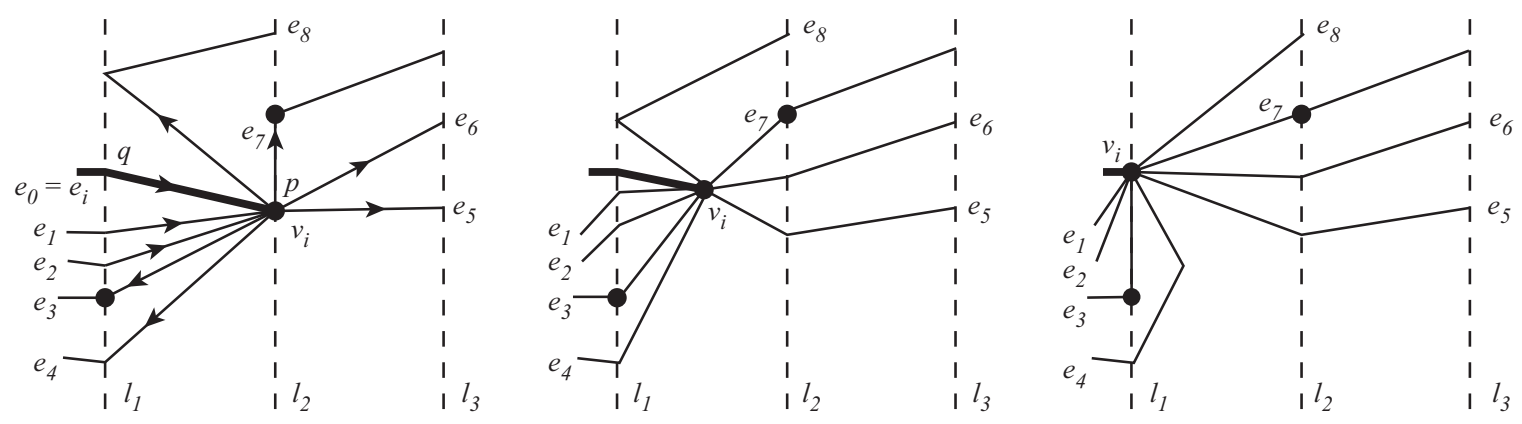

Figure 1: The main step of the algorithm moves $v_{i}$ along one segment of $e_{i}$.

The operation is local, altering only the position of $v_{i}$ and of bends and vertices joined to $v_{i}$ by a straight segment. These bends and vertices all lie on line $l=l_{2}$, its predecessor $l_{1}$ and its successor $l_{3}$. Since bends and vertices only occur on vertical lines, portions of edges between adjacent vertical lines are straight, which makes it possible to use linear morphs. By Property (1) all incoming edges to $v_{i}$ are contiguous and arrive from the same side; assume without loss of generality that they arrive from $l_{1}$. Also by Property (1) there are no vertices/bends along $l_{1}$ between the incoming edges. The situation is as shown in Figure 1. We morph as follows:

vertex $v_{i}$ : Move vertex $v_{i}$ along $e_{i}$ from its current point $p$ on $l_{2}$ to point $q$ where $e_{i}$ intersects $l_{1}$ 
incoming edges: These arrive from bends on $l_{1}$. Move these bends along $l_{1}$ to $q$. (See edges $e_{1}$ and $e_{2}$ in Fig. 1.)

outgoing edges: There are several cases: Any edge to $l_{3}$ acquires a new bend on $l_{2}$, initially at $p$, and with final positions nicely spaced on $l_{2}$. (See $e_{5}$ and $e_{6}$.) There may be an edge to a vertex on $l_{2}$-leave the vertex fixed. (See $e_{7}$.) Finally, consider an edge to a bend/vertex $t$ on $l_{1}$. If $t$ is a bend, and the interval along $l_{1}$ between $q$ and $t$ contains only bends connected to $v_{i}(*)$ then move $t$ to $q$. (See $e_{8}$.) If $t$ is a vertex and property $(*)$ holds then $t$ stays fixed and the edge $\left(v_{i}, t\right)$ morphs to lie along $l_{1}$. (See $e_{3}$.) Otherwise $(*)$ fails which means that there is an intervening bend/vertex along $l_{1}$ between $q$ and $t$. In this case $t$ stays fixed and the segment $\left(v_{i}, t\right)$ morphs to a two-segment path that bends around the intervening point(s). (See $e_{4}$.) We defer further details and justification of planarity to the full paper.

Each main step of the algorithm can be accomplished via two linear morphs, the dividing point being when the new bends appear.

\section{Analysis}

Note that the final case of the main step of the algorithm (see the right-hand pane of Fig. 1) introduces new bends. Each bend requires a new vertical line, and each crossing of the vertical line with an existing edge counts as a new bend. This blow-up in the number of bends is potentially dangerous, since the number of main steps performed by the algorithm depends on the number of bends. To circumvent the danger, we focus on turns, which are bends where the path changes $x$-direction, and we examine more closely the straightening step and the main step of the algorithm, and argue that turns are progagated rather than created. Looking at the big picture, the intuition is that, since iteration $i$ of the algorithm causes each outgoing edge of $v_{i}$ to follow the path of the incoming edge $e_{i}$, thus, at worst, we copy the turns of $e_{i}$ onto all the outgoing edges. We begin by bounding the number of turns introduced during the set-up phase. Further details of the following theorem are defered.

Theorem 1 The algorithm uses $O\left(n^{6}\right)$ linear morphs.

\section{References}

[1] S. S. Cairns. Deformation of plane rectilinear complexes. American Mathematical Monthly, 51:247-252, 1944.

[2] H. de Fraysseix, J. Pach, and R. Pollack. How to draw a planar graph on a grid. Combinatorica, 10(1):41-51, 1990.

[3] M. S. Floater and C. Gotsman. How to morph tilings injectively. Journal of Computational and Applied Mathematics, 101:117-129, 1999.

[4] C. Gotsman and V. Surazhsky. Guaranteed intersection-free polygon morphing. Computers and Graphics, 25:67-75, 2001.

[5] W. Schnyder. Embedding planar graphs on a grid. In Proceedings of the ACM-SIAM Symposium on Discrete Algorithms, pages 138-148, 1990.

[6] C. Thomassen. Deformation of plane graphs. Journal of Combinatorial Theory Series B, $34: 244-257,1983$. 